\title{
Role of the locus coeruleus arousal system in cognitive control
}

\author{
Grueschow, Marcus ; Kleim, Birgit ; Ruff, Christian C
}

\begin{abstract}
Cognitive control lies at the core of human adaptive behaviour. Humans vary substantially in their ability to execute cognitive control with respect to optimally facing environmental challenges, although the neural origins of this heterogeneity are currently not well understood. Recent theoretical frameworks implicate the locus coeruleus noradrenergic arousal system (LC-NE) in that process. Invasive neurophysiological work in rodents has shown that the LC-NE is an important homeostatic control centre of the body. LC-NE innervates the entire neocortex and has particularly strong connections with the cingulate gyrus. In the present study, using a response conflict task, functional magnetic resonance imaging and concurrent pupil dilation measures (a proxy for LC-NE firing), we provide empirical evidence for a decisive role of the LC-NE in cognitive control in humans. We show that the level of individual behavioural adjustment in cognitive control relates to the level of functional coupling between LC-NE and the dorsomedial prefrontal cortex, as well as dorsolateral prefrontal cortex. Moreover, we show that the pupil is substantially more dilated during conflict trials requiring behavioural adjustment than during no conflict trials. In addition, we explore a potential relationship between pupil dilation and neural activity during choice conflict adjustments. Our data provide novel insight into arousal-related influences on cognitive control and suggest pupil dilation as a potential external marker for endogenous neural processes involved in optimising behavioural control. Our results may also be clinically relevant for a variety of pathologies where cognitive control is compromised, such as anxiety, depression, addiction and post-traumatic stress disorder.
\end{abstract}

DOI: https://doi.org/10.1111/jne.12890

Posted at the Zurich Open Repository and Archive, University of Zurich

ZORA URL: https://doi.org/10.5167/uzh-191584

Journal Article

Accepted Version

Originally published at:

Grueschow, Marcus; Kleim, Birgit; Ruff, Christian C (2020). Role of the locus coeruleus arousal system in cognitive control. Journal of Neuroendocrinology, 32(12):e12890.

DOI: https://doi.org/10.1111/jne.12890 


\title{
Title Page
}

Submission as Neuroendocrinology and Brain Imaging Special Issue

Contribution:

\section{Role of the Locus Coeruleus Arousal System in}

\section{Cognitive Control}

\author{
Marcus Grueschow ${ }^{1 *}$, Birgit Kleim ${ }^{2,3}$, Christian C. Ruff ${ }^{1}$
}

${ }^{1}$ Zurich Center for Neuroeconomics (ZNE), Department of Economics, University of Zurich, Zurich, Switzerland

${ }^{2}$ Department of Experimental Psychopathology and Psychotherapy, University of Zurich, Zurich, Switzerland

${ }^{3}$ Department of Psychiatry, Psychotherapy and Psychosomatics, University of Zurich, Zurich, Switzerland

* Corresponding authors:

Dr. Marcus Grueschow, Department of Economics, University of Zurich, 8006 Zurich, Blümlisalpstrasse 10, Switzerland. marcus.grueschow@econ.uzh.ch

Key Words: response conflict adjustment, executive function, arousal, pupil dilation, functional connectivity, DMPFC, ACC, DLPFC

Short title: LC-NE in Cognitive Control 


\begin{abstract}
Cognitive control lies at the core of human adaptive behaviour. Humans vary substantially in their ability to execute cognitive control in order to optimally face environmental challenges, but the neural origins of this heterogeneity are currently not well understood. Recent theoretical frameworks implicate the locus coeruleus noradrenergic arousal system (LC-NE) in that process. Invasive neurophysiological work in rodents has shown that the LC-NE is an important homeostatic control centre of the body. LC-NE innervates the entire neo-cortex, and has particularly strong connections with the cingulate gyrus. Here, using a response conflict task, fMRI, and concurrent pupil dilation measures (a proxy for LC-NE firing), we provide empirical evidence for a decisive role of the LC-NE in cognitive control in humans: We show that the level of individual behavioural adjustment in cognitive control relates to the level of functional coupling between LC-NE and the dorso-medial prefrontal-cortex (DMPFC) as well as dorso-lateral prefrontal cortex (DLPFC). Moreover, we show that the pupil is substantially more dilated during conflict trials requiring behavioural adjustment than during no conflict trials. In addition, we explore a potential relationship between pupil dilation and neural activity during choice-conflict adjustments. Our data provide novel insight into arousal-related influences on cognitive control and suggest pupil dilation as a potential external marker for endogenous neural processes involved in optimizing behavioural control. Our results may also be clinically relevant for a variety of pathologies where cognitive control is compromised such as anxiety, depression, addiction and PTSD.
\end{abstract}




\section{Introduction}

Cognitive control is the ability to flexibly shape and constrain thoughts and actions in service of accomplishing internal goals ${ }^{1}$. A crucial aspect of cognitive control is the resolution of response conflict, which is essential for decision making and adaptive behaviour ${ }^{2,3}$. Response conflict arises when a pre-potent habitual response is suppressed for the choice of an alternative option that better fits present behavioral goals ${ }^{1,4}$. Humans vary substantially in the ability to resolve response conflict, but the neural origins of this heterogeneity are currently not well understood.

The prominent conflict-monitoring model of cognitive control assigns a central role to the dorso-medial prefrontal cortex (DMPFC). Classic work has shown that this region, spanning from anterior cingulate cortex (ACC) to the supplementary motor area (SMA), detects and monitors the level of behavioral conflict ${ }^{5-8}$. In this framework, DMPFC communicates this information to the dorso-lateral prefrontal cortex (DLPFC), which subsequently implements appropriate adjustments and resolves the conflict through cortical amplification of taskrelevant information ${ }^{1,9,10}$. An early extension of this model speculated that noradrenergic arousal processes may play a crucial role in cognitive control and response conflict adjustments 11-13. In addition, recent behavioral and pupillometry work suggested that variation in the functioning of the locus coeruleus-norepinephrine (LC-NE) arousal system may account for individual differences in cognitive control capacity ${ }^{14,15}$. However, to date very little neurophysiological evidence in humans associates the LC-NE arousal system with individual differences in conflict resolution. Just one previous fMRI study has so far suggested that different human brainstem nuclei may be activated during cognitive control ${ }^{16}$.

In animals, tracing studies have provided evidence for anatomical ${ }^{17-19}$ and functional connections between the conflict monitoring DMPFC and the LC-NE ${ }^{20-23}$. In addition, the 
region mostly associated with conflict resolution, the DLPFC ${ }^{24,25}$, is also well interconnected with regions in the DMPFC such as premotor cortex and via reciprocal connections with SMA and pre-SMA ${ }^{26}$. However, there is only limited knowledge as to how the functional coupling between DMPFC, DLPFC the LC-NE, and other connected regions relate to the individual capacity for behavioral adjustments.

An individual's capacity for conflict resolution is typically quantified via reaction time differences between conflict trials, which require the resolution of conflict and no conflict trials during which no such resolution is necessary ${ }^{1,5,6}$. The resolution of conflict is thought to incur processing costs due to the necessity to detect, monitor and finally adjust the behavioural conflict, which eventually results in longer reaction times for conflict as compared to no conflict trials. Importantly, the better an individual's capacity to resolve the behavioural conflict, the smaller these reaction time differences. In other words, better regulators of behavioural conflict exhibit smaller differences in reaction times between these two trial types. Here we use this reaction time difference score to quantifiy an individual's regulatory capacity and relate it to the functional coupling of DMPFC in order to provide neurophysiological evidence for the observed individual differences in behavior. In particular, we hypothesize that the locus coeruleus noradrenergic system (LC-NE) may play a crucial role in response conflict resolution and may underlie individual differences in capacity for cognitive control, as previously speculated ${ }^{14}$. For such a decisive role, the locus coeruleus arousal system is indeed in a unique functional position, as it innervates the entire neo-cortex with noradrenergic projections and has particularly strong connections with the conflict monitoring DMPFC ${ }^{17,18,20}$. Moreover its hypothalamic connections and responsivity to emotional and physiological stress indicate that it may play a critical role in regaining homeostasis and optimizing behaviour 27-29. The LC-NE is also clinically relevant for a variety of pathologies in which cognitive control is compromised such as anxiety, depression, addiction and PTSD ${ }^{30-37}$. 
In addition to non-invasively measuring functional imaging data, we concurrently track variations in pupil size during congnitive control behaviour as LC-NE firing has been related to pupil dilation (Joshi et al. 2016). With this measure, we aim to establish a link between the cheap and easy acquired external pupil measures during response conflct resolution and neural activity in regions previously related to cognitive control ${ }^{6,24,38}$.

\section{Material and Methods}

Participants. We recruited 48 medical students ( $n=28$ women, mean age $=24$ years, $S D=1.99$ ) following standard exclusion criteria (fMRI safety, psychopathology). Participation was voluntary and participants provided written informed consent. After the study, participants were debriefed and compensated for their participation (CHF 35 per hour, which roughly corresponds to 35 US\$). All procedures were approved by the Cantonal Ethics Committee of Zurich (KEK).

Stimulus presentation. All stimuli were displayed on a grey projection screen (using the Cogent2000-toolbox, http://www.vislab.ucl.ac.uk/cogent_2000.php, implemented in Matlab, The MathWorks, Inc., Natick, Massachusetts, United States) that participants viewed by means of a mirror system mounted atop the MR head coil. The task comprised 50 congruent and 50 incongruent trials, presented in pseudorandom order and counterbalanced for equal numbers of congruent-congruent, congruent-incongruent, incongruent-congruent, and incongruentincongruent temporal stimulus pairings (however, please note that we only focus on simple congruency effects in this work and describe the trialwise sequence effects in another manuscript). Most participants conducted two runs of the emotional Stroop task (46 out of 48), which amounts to 200 trials. Two participants only did one run which amounts to 100 trials. The intertrial intervals (ITI) for each participant were individually sampled from a gamma distribution using the matlab function gamrnd.m (Matlab, Mathworks) with shape parameter 2 
and scale parameter 1 and truncated within 2 and 6 seconds, to optimally spread 100 trials across 10 min of one functional run time. This yielded a mean ITI of $3.1 \mathrm{sec}$. To avoid any priming effects, there were neither direct repetitions of the same face with varying word distracters nor direct repetitions of exact face-word-distracter combinations ${ }^{39,40}$. Genders, identities, and affective expressions on the faces were randomized throughout the task and stimulus occurrences were counterbalanced across trial types and response buttons. Subjects were instructed to respond as fast as possible to the stimulus by pressing one of two buttons (left: happy, right: fear or vice-versa) on an MR-compatible response box, while trying to maintain high accuracy. In addition, each participant conducted 1-2 runs of an attentional capture task (10min per run, these data will be reported elsewhere).

Behavioral Task. We employed the emotional-Stroop task ${ }^{38,41,42}$, a well-established laboratory measure of conflict ${ }^{43,44}$. Participants categorized faces according to their emotional expression (happy vs. fearful) while at the same time ignoring overlayed emotionally congruent (C) or incongruent (I) words ("HAPPY", "FEAR", Figure 1A-B). Behaviorally, the conflict is typically observed as higher reaction times (RT) for incongruent than congruent trials $38,41,45$ (Figure 1C).

(Figure 1 about here)

Behavioral analyses. Behavioral data consisted of both reaction times (excluding error and post-error trials) and accuracy rates. A response was considered correct when the emotional valence of the face expression was correctly identified. Trials with response times above 2 standard deviations from the mean (across all trials) were excluded from analysis (and regarded as trials of no interest in the fMRI-models ${ }^{38,41,46}$, see below). Statistical behavioral analyses were performed using paired t-tests implemented in the statistics toolbox in matlab comparing conflict (I) vs. no conflict trials (C). An individual score for response conflict resolution was 
computed as the reaction time difference between congruent and inconguernt trials (Figure 1C).

fMRI image acquisition. Subjects performed two fMRI sessions of the emotional stroop task, each lasting 9.75 minutes. During each session, we acquired $225 \mathrm{~T} 2 *$-weighted whole-brain echo planar images using a Philips Achieva 3 T whole-body scanner (Philips Medical Systems, Best, The Netherlands) equipped with an 8-channel Philips sensitivity-encoded (SENSE) head coil. Imaging parameters were: $2600 \mathrm{~ms}$ repetition time (TR); $40 \mathrm{~ms}$ (TE); 37 slices (transversal, ascending acquisition); $2.6 \mathrm{~mm}$ slice thickness; $2.5 \mathrm{~mm} \times 2.5 \mathrm{~mm}$ in-plane resolution; $0.65 \mathrm{~mm}$ gap; $90^{\circ}$ flip angle. To measure at fully equilibrated magnetic field, five dummy-image excitations were performed and discarded before functional image acquisition started. Additionally, we acquired a high-resolution, T1-weighted 3D fast-field echo structural scan used for image registration during post-processing (sequence parameters: 181 sagittal slices; matrix size: 256 x 256; voxel size: 1 x 1 x 1 mm; TR/TE/TI: 8.3/2.26/181 ms).

fMRI image pre-processing. Image preprocessing and analysis were conducted using SPM8 (Wellcome Trust Centre for Neuroimaging). Functional images were slice-time corrected (to the middle slice acquisition time) and realigned (accounting for subjects' head motion). Each subjects' T1-weighted structural image was co-registered to the mean functional image and normalized to the standard T1-MNI template using the "Unified Segment" procedure provided by SPM8 ${ }^{47}$. The functional images were then normalized to the standard MNI template using the same transformation, spatially resampled to $2.5 \mathrm{~mm}$ isotropic voxels, and smoothed using a Gaussian kernel (FWHM, 6mm).

fMRI data-analysis. We estimated a general linear model (GLM) to identify regions associated with conflict processing, defined as the BOLD activation difference between I and C trials. This contrast was used to test whether any region covaried with the individual response conflict resolution score defined as the reaction time difference between congruent and inconguernt 
trials (Figure 1C). The smaller the RT difference between these two trial types, the better the level of response conflict resolution of the participant. The GLM contained two indicator functions placed at the onset of each of the possible trial types (congruent and incongruent). An additional indicator function modelled the onsets for trials of no interest, which included: trials with reaction times 2 standard deviations above the participant's overall mean response time as well as error and post-error trials that may be associated with error-related cognitive processing ${ }^{38,41}$. The GLM regressed the blood-oxygen level-dependent (BOLD) signal in each voxel on these regressors and a set of hyperparameters modelling MR image auto-correlations with a first-order autoregressive model. Six motion parameters (obtained during the realignment procedure) were also included as regressors of no interest to account for participants' head motion. Furthermore, we included additional regressors that accounted for variance induced by eye-related variables (blinks and saccades, see below), to ensure that neural conflict responses are not confounded by these variables ${ }^{48}$. The model thus included additional indicator functions for the onsets of blinks and saccades.

First-level summary statistics were obtained by calculating the single-subject voxel-wise contrasts of incongruent $>$ congruent trials (I $>C$, quantifying conflict) (Figure 2). Statistical inference was performed with a random-effects General Linear Model within the SPM8 framework. The whole-brain FWE-corrected statistical threshold was set to $P<0.05$ with an initial cluster-defining voxel-level threshold of $\mathrm{T}=3.275$ (equivalent to uncorrected $P<0.001$ ) ${ }^{49,50}$. For hypothesis-guided ROI analysis of the LC-NE arousal system and DLPFC, we applied small volume peak-level FWE-correction restricted to a 2 SD-locus coeruleus volume mask ${ }^{51}$ and a $10 \mathrm{~mm}$ radius sphere centered around DLPFC coordinates reported by ${ }^{16}$ for the $\mathrm{I}>\mathrm{C}$ contrast $(X, Y, Z=48,10,36)$.

Psychophysiological analysis (PPI). We added to our GLM design matrix the BOLD timeseries extracted from a 5mm sphere centered on the DMPFC-peak (X/Y/Z: -7/13/55) identified 
with the incongruent $>$ congruent contrast. We also added two interaction terms corresponding to the interactions of the extracted BOLD time-course and the $\mathrm{C}$ and I regressors. Note that these interaction terms cannot be confounded by the main effects and interactions of all other experimental variables, which were accounted for by the regressors in the original design matrix. In a second-level analysis, the difference in functional coupling during conflict processing $(\mathrm{I}>\mathrm{C})$ was correlated across the whole brain with the response conflict resolution score (RT difference between I $>$ C) (Figure 3).

Eye measures. During scanning, eye movements were sampled at $250 \mathrm{~Hz}$ using an MRcompatible infrared EyeLink II CL v4.51 eye-tracker system (SR Research Ltd.). In order to account for the effects of eye movements and blinks on BOLD responses, we added them as regressors of no interest to the general linear model (see above). Saccades were defined as eye movements larger than 0.5 degrees visual angle ${ }^{52}$. Blinks were defined as periods of signal loss lasting longer than $80 \mathrm{~ms}$ and shorter than $2000 \mathrm{~ms}{ }^{53}$; these epochs were removed from the pupil data and filled in by linear interpolation.

Pupil dilation. Pupil time-series were high-pass-filtered with a low cut-off of $0.05 \mathrm{~Hz}$ followed by a low-pass-filter with a high cut-off of $4 \mathrm{~Hz}$. Each run-wise pupil time-series was z-scored. Trial-wise pupil data were extracted \pm 5 seconds from stimulus onset and averaged per subject according to conditions of interest (congruent, incongruent Figure 4 A-B). The contrast incongruent $(\mathrm{I})>$ congruent $(\mathrm{C})$ was computed for each participant. Time periods of significant difference from zero $(\mathrm{p}<0.05)$ across all participants were identified via one-sample-ttests versus zero (Figure 4 B) and corrected for multiple comparisons using a cluster-based permutation test (see below). For each participant, the I $>$ C pupil dilation within the significant time intervals (grey shading Figure 4B) was averaged to derive one pupil dilation difference score per subject, reflecting the level of individual phasic noradrenergic tone ${ }^{54-56}$ during response conflict resolution. This individual pupil score $(I>C)$ was added as covariate in a $2^{\text {nd }}$ 
level analysis of individual BOLD contrasts $(\mathrm{I}>\mathrm{C})$. This analysis identifies brain regions in which the $\mathrm{I}>\mathrm{C}$ difference is reflected in the $\mathrm{I}>\mathrm{C}$ difference in pupil dilation and for which pupil dilation may thus be an external read-out (Figure 4).

Pupil dilation cluster-correction. To identify time windows during which the pupil dilation significantly differs between incongruent and congruent trials, while avoiding false positive clusters, we applied Bonferroni-correction ${ }^{57,58}$ via a cluster-based permutation test following 49. We used a cluster-forming threshold of $\mathrm{T}=2.02$ corresponding to a two-sided $\mathrm{p}$-value of 0.05 given 47 degrees of freedom $(\mathrm{N}=48)$. The procedure first calculates the one-sample tstatistic across all participants' average difference between relevant contrasts for each 4millisecond bins in the pupil dilation time series. Next, the size of continuous temporal clusters, defined as the number of adjacent time bins exceeding the cluster-forming threshold, were identified and tested against cluster sizes observed by chance. To this end, a null distribution of cluster sizes was generated by permuting the labels for each trial and time bin withinparticipant by flipping the sign of each time bin randomly 1000 times and recomputing the tstatistic across all time bins for each iteration. On each iteration the largest permuted temporal cluster was identified and stored in the null distribution. A cluster corrected p-value is computed by dividing the number of clusters in the null distribution exceeding the number of clusters in the data by the number of iterations.

\section{Results}

\section{DLPFC relates to level of response conflict resolution}

Our results replicate classic behavioral and neural effects of response conflict ${ }^{6,24,38}$. For instance, individuals are generally slower to respond to incongruent trials ${ }^{45}$. Comparing incongruent (conflict) with congruent (no conflict) trials, we found significantly increased RTs 
$\left(T_{47}=9.88, p=4.67 * 10^{-13}\right.$, Figure 1C) and decreased accuracy $\left(T_{47}=-5.25, p=3.65 * 10^{-6}\right.$,

Figure 1D). Theoretical frameworks ${ }^{5}$ and empirical findings ${ }^{6,24,38}$ predict that the monitoring and processing of conflict is reflected in activity of the dorsomedial prefrontal (DMPFC) and anterior cingulate cortex (ACC). Thus, not surprisingly, the $\mathrm{I}>\mathrm{C}$ contrast revealed the dorsomedial prefrontal cortex $\left(\mathrm{DMPFC}, \mathrm{P}_{(\mathrm{FWE})}=0.032, \mathrm{X} / \mathrm{Y} / \mathrm{Z}:-7 / 13 / 55\right)$ extending into anterior cingulate cortex, a region strongly associated with response conflict in several prior studies ${ }^{6,24,38}$ (Figure 2A).

In order to identify the neurophysiological origin of the observed individual differences in behavior, we correlated each participant's reaction time difference score $(I>C)$ with the corresponding differences in BOLD activity (Figure 2B) observed between conflict versus no conflict trials $(\mathrm{I}>\mathrm{C})$. As hypothesized, we found that the individual level of response conflict resolution was related to DLPFC activation strength $\left(\mathrm{T}_{(\mathrm{FWE}-\mathrm{SVC})}=3.26, \mathrm{P}=0.041, \mathrm{X} / \mathrm{Y} / \mathrm{Z}\right.$ : 43/18/35). More specifically, the smaller the reaction time difference between conflict as compared to no conflict trials, the stronger the difference in BOLD activity between conflict versus no conflict trials in DLPFC. In other words, the stronger the DLPFC activity difference between conflict versus no conflict trials, the better the participants were able to adjust their behavior (Figure 2B).

(Figure 2 about here)

\section{Relating DMPFC functional coupling to individual level of response conflict resolution}

In order to establish a behaviorally-relevant link also between functional connectivity in the conflict monitoring region (DMPFC) and the rest of the brain, we again use the reaction time difference score $(\mathrm{I}>\mathrm{C})$ for each participant and correlated it with the differences in functional coupling of DMPFC during conflict versus no conflict trials $(\mathrm{I}>\mathrm{C})$ (Figure 3A). We found that the level of behavioral choice conflict adjustment is strongly reflected in the functional 
coupling between DMPFC and DLPFC $\left(\mathrm{T}_{(\mathrm{FWE})}=5.14, \mathrm{P}=0.017, \mathrm{X} / \mathrm{Y} / \mathrm{Z}: 43 / 31 / 35\right.$, Figure 3B) as well as between DMPFC and the bilateral locus coeruleus noradrenergic system (right LC: $\mathrm{T}_{(\mathrm{FWE}-\mathrm{SVC})}=3.43, \mathrm{P}=0.005, \mathrm{X} / \mathrm{Y} / \mathrm{Z}: 6 /-37 /-23$, left LC: $\mathrm{T}_{(\mathrm{FWE}-\mathrm{SVC})}=3.21, \mathrm{P}=0.009$, $\mathrm{X} / \mathrm{Y} / \mathrm{Z}$ : -5/-37/-23, Figure 3C). Interestingly, we also found two additional clusters for which the connectivity difference between $\mathrm{I}>\mathrm{C}$ was related to response conflict resolution performance: the fusiform face area (right FFA: $\mathrm{T}_{(\mathrm{FWE})}=4.77, \mathrm{P}=0.008, \mathrm{X} / \mathrm{Y} / \mathrm{Z}: 53 /-52 /-18$ ) as well as the intraparietal sulcus (right IPS: $\mathrm{T}_{(\mathrm{FWE})}=4.55, \mathrm{P}=0.046, \mathrm{X} / \mathrm{Y} / \mathrm{Z}: 23 /-65 / 38$ ). These results indicate an enhancement of functional coupling between the conflict-monitoring DMPFC with regions representing task-relevant variables, in our case faces in the FFA. These findings substantiate the notion that neural representation of task-relevant variables are actively enhanced to help resolve behavioural conflict, which directly leads to enhanced performance, i.e., better behavioural adjustments.

(Figure 3 about here)

\section{Exploring individual differences in neural activity during conflict processing to individual differences in pupil dilation}

Given a previously established link between LC-NE firing and pupil dilation (but see Reimer et al. for a cholinergic account), we tested the hypothesis that differences in pupil dilation during the response conflict resolution process may also relate to neural activity in regions previously associated with cognitive control such as DMPFC or DLPFC or even with the locus coeruleus. We first investigated whether pupil dilation differs between conflict and no conflict trials. We found substantially enhanced pupil dilation in conflict versus no conflict trials within a time-window of 1 to ca 3.5 seconds post stimulus onset (Figure 4A-B), suggesting a potential noradrenergic contribution to choice conflict adjustment (continuous cluster between $945 \mathrm{~ms}$ to $3668 \mathrm{~ms}, \mathrm{p}<0.05$, one-sample t-test, corrected for multiple comparisons using cluster-based permutation test, two-sided, $\mathrm{df}=47$, please see methods for details). Additionally, we correlated the individual pupil dilation difference $(\mathrm{I}>\mathrm{C})$ during the identified significant time window with the BOLD activity difference $(\mathrm{I}>\mathrm{C})$. We did not find any region surviving our 
stringent whole brain family-wise error correction criterion. However, for completeness we report here the biggest uncorrected cluster that related to pupil dilation differences $(I>C)$, which was located in the anterior cingulate cortex $\left(\mathrm{ACC}: \mathrm{T}_{\text {(uncorected) }}=3.25, \mathrm{P}=0.001, \mathrm{X} / \mathrm{Y} / \mathrm{Z}: 6 / 13 / 25\right.$, $\mathrm{k}=53$, Figure 4C).

(Figure 4 about here)

\section{Discussion}

A crucial aspect of human adaptive behaviour and decision-making is the resolution of response conflict. Humans vary substantially in the ability to resolve response conflict, but the neural origins of this heterogeneity are currently not well understood. Recent theoretical frameworks have proposed that noradrenergic arousal plays a role in this process, but little neurophysiological evidence links individual functioning of the human LC-NE arousal system to an individual's ability to resolve response conflict ${ }^{14-16}$. Here we provide such evidence. More specifically, we show that the level of individual conflict resolution (reaction time differences between conflict trials and no-conflict trials) is strongly related to the level of functional coupling between the conflict-monitoring region DMPFC and the LC-NE arousal system as well as the DLPFC. The more effective the conflict resolution, the stronger the functional coupling between these regions. Moreover, we show that pupil dilation is substantially enhanced during response conflict resolution as compared when no resolution is required. The presented data provide novel insights into the role of arousal systems for determining the individual ability to deal with response conflict and the capacity for cognitive control ${ }^{1}$.

The classic conflict-monitoring theory of cognitive control posits that the anterior cingulate cortex (ACC) in the DMPFC serves the essential role of detecting and tracking behaviourally relevant levels of conflict that require resolution ${ }^{1,6,24}$. It has further been proposed that ACC 
activity may serve as a signal that engages regulatory processes in the DLPFC to implement performance adjustments ${ }^{10,59}$, suggesting that increased DLPFC activity during cognitive control performance may be related to beneficial behavioral control. Much to our surprise, very little data exists that link individual differences in conflict resolution performance to activity levels in the DLPFC or to the level of functional connectivity within these proposed cognitive control circuits ${ }^{1}$. Neverthless, our data support the classic model by revealing that participants with increased activity in the DLPFC during conflict resolution also exhibited enhanced response conflict adjustment performance. In addition, the relevance of the DLPFC in behavioural control performance is further corroborated by our functional connectivity data. We show that the stronger the functional connectivity between the monitoring region DMPFC and the regulation region DLPFC during behavioral conflict resolution, the better the cognitive control performance (i.e., the smaller the reaction time differences $(I>C))$. The same network was identified in a previous study investigating ACC/DMPFC functional connectivity during conflict processing with comparable picture-word interference stimuli as employed here ${ }^{60}$. Congruent with our results, the authors also found stronger functional connectivity between an ACC seed region and DLPFC in conflict vs. no conflict trials. Moreover, the importance of this network for behavioural control in general is supported by other studies of cognitive control with other modalities. For instance, using the stop-signal task, its been shown that the DMPFC shows greater activation during stop error as compared to go success trials, and that DLPFC shows greater activation during post-error go trials with RT slowing as compared to post-error go trials without RT slowing ${ }^{61}$. Moreover, electrophysiological evidence has also implicated this network in behavioral adjustment for proactive control ${ }^{62}$.

The role of DLPFC in the conflict monitoring model is classically thought to engage in regulatory processes to implement performance adjustments depending on the level of conflict detected by the DMPFC ${ }^{10,59}$. It is further assumed that these regulatory processes particularly 
impact on task-relevant regions ${ }^{9}$ to enhance neural coding of task-relevant variables, thereby improving conflict resolution. In the emotional-stroop-task as employed here, participants are asked to focus on and report the emotion of a face while disregarding word distractors. In accordance with predictions from the conflict monitoring model, we find that during conflict trials, functional coupling between the face selective fusiform face area ${ }^{63-65}$ and the DMPFC directly relates to the individual conflict adjustments performance. This result suggests that enhanced communication between monitoring and perceptual regions can substantially improve task performance and potentially also cognitive control as shown here. However, please note that our data cannot resolve whether the information regarding the level of conflict that requires resolution must first pass through the DLPFC ${ }^{1,6,24}$.

Enhancing activity of regions coding task-relevant variables is a function also assigned to the locus coeruleus noradrenergic system (LC-NE) ${ }^{66}$, but the role of LC-NE in tasks requiring active cognitive control has remained underexplored. Nevertheless, while resting-state fMRI studies have demonstrated LC-NE functional connectivity with brain regions involved in executive control ${ }^{67,68}$, an active noradrenergic role in conflict processing and resolution has so far mainly been suggested ${ }^{11}$, primarily based on recent behavioural and pupil dilation evidence using various types of paradigms involving cognitive control ${ }^{14-16}$.

For instance, multiple studies have shown that across three cognitive control domains (updating, switching and inhibition), increases in task demands typically lead to increases in pupil dilation ${ }^{69}$. Specifically in Stroop interference tasks, multiple studies have previously reported increased pupil dilation in conflict (versus no conflict) trials, which are typically associated with increased effort required to resolve the conflict ${ }^{69-73}$. Please see van der Wel et al. 2018 for an excellent literature review on pupil dilation in cognitive control, clearly identifying pupillometry as a useful and robust neurophysiplogical research tool ${ }^{69}$. In addition, an increasing body of evidence has started to reveal the brain mechanisms that underlie effort- 
related pupil dilation and has primarily shown correlations of noradrenergic locus coeruleus activity with pupil dilation ${ }^{27,74,75}$, but also with ACC activity ${ }^{74}$.

The notion that LC-NE activity enhances task performance on a multitude of perceptual tasks is longstanding and particularly grounded in rodent neurophysiology ${ }^{76-81}$. For instance, it has been shown that higher NE concentration in the rodent brain is associated with improvements in sensory encoding ${ }^{76,82,83}$, better signal detection performance ${ }^{78,84}$, and eventually increased sensory discrimination ${ }^{85-87}$. Moreover, heightened noradrenergic tone has been linked with faster and more accurate perceptual choices in humans and non-human primates $55,56,78,79,88$. Our findings now extend this performance-enhancing role of LC-NE activity to the behavioural domain of executive functions involving conflict resolution and cognitive control. We show that functional coupling between the conflict-monitoring region DMPFC and LC-NE during conflict adjustments is directly related to behavioural performance. These findings may suggest that depending on the level of conflict detected by DMPFC, the LC-NE arousal system may also help upregulate activity in regions coding task-releavant variables, similarly to the prescribed role for DLPFC. This interpretation accords with a previous finding that functional connectivity between LC-NE seed region and both DLPFC as well as ACC was enhanced during conflict trials as compared to no conflict trials ${ }^{16}$.

These previous human LC-NE connectivity reports, as well as animal neurophysiological tracer studies, also directly link with our pupil dilation results, as these studies have indicated a prominent anatomical and functional connectivity between LC-NE and DMPFC and particularly the ACC ${ }^{17,18,20}$. Here we find much larger pupil dilations during conflict trials as compared to no conflict trials. Given the proposed link between LC-NE firing and pupil dilation, these findings suggest a noradrenergic contribution to response conflict resolution, as previously suggested $11,69,89-91$, thereby corroborating theoretical considerations ${ }^{11-15}$. In addition, even though not surviving stringent cluster correction, the location of the ACC region 
relating to pupil dilation differences $(\mathrm{I}>\mathrm{C})$ in our results corresponds to a region that has previously been associated with unsigned reward prediction error ${ }^{92,93}$. This is in line with the notion that pupil signals may reflect uncertainty and a behavioural surprise component that is highly salient and arousing ${ }^{94,95}$.

Our study is not without limitations. Due to its small size and proximity to the ventricles functional imaging of the brainstem is difficult ${ }^{96-98}$. It is therefore not entirely clear whether our imaging results in the brainstem only reflect LC-NE activity or activity from neighbouring areas. Future studies may address this problem with more specialized imaging protocols. For instance, to enhance signal-to-noise ratio, high-field imaging at 7 Tesla in combination with partial brain coverage would allow particularly small (submillimeter) voxel resolution and reduce partial voluming effects in small structures of the brain and be less susceptible to pulsating artefacts from the adjacent 4th ventrical. Moreover, a T1-TSE sequence would allow direct and individual identification of the LC-NE via neuromelanin-sensitive contrast $51,99,100$. Neuromelanin-sensitive sequences are also clinically relevant and may provide substantial translational value. Previous studies using these imaging techniques have highlighted potential pathology of the LC circuit in neuropsychiatric conditions associated with deficits in cognitive control, such as for example in Alzheimer's and Parkinson's disease ${ }^{101}$. In addition, altered LC integrity in pathological aging has also been identified using these innovative sequences ${ }^{102}$. Irrespective of such considerations, the convergence of our imaging and pupil results provides important neurophysiological evidence that characteristics of an individual's LC-NE system may be one of the subcortical neuro-modulatory systems contributing to the capacity for cognitive control.

In conclusion, our data provide insights into the role of arousal systems for determining the individual ability to deal with response conflict. Moreover, our results establish pupil dilation as a valuable external marker for endogenous neural processes involved in adjusting and 
Grueschow et al. - LC-NE in Cognitive Control

optimizing behaviour. These findings may be clinically relevant for a variety of pathologies with impairments of cognitive control, such as anxiety, depression, addiction and PTSD. 
Grueschow et al. - LC-NE in Cognitive Control

\section{Acknowledgments}

The authors would like to thank Karl Treiber for scanning assistance and Adrian Etter and Marc Biedermann for support with the eye-tracking setup. This research was funded by grants awarded by the Swiss National Science Foundation to B.K. (PZ00P1_126597, PZ00P1_150812) and C.C.R. (100019L_173248). M.G. was funded by a grant awarded by the Richard-Büchner-Foundation (F-33153-02-01).

\section{Author Contributions}

M.G., B.K., and C.C.R. conceived of the project. M.G., C.C.R. and B.K. designed the study. M.G. collected the data. M.G. performed all analyses. M.G., C.C.R. and B.K. wrote the manuscript. All authors discussed the results and contributed to the final manuscript.

\section{Competing Interests statement}

The authors declare no competing interests.

\section{Data Availability}

The raw behavioral data will be made available on the data sharing repository: https://osf.io/

\section{Code Availability}

The experimental code will be made available at: https:/github.com/mgrues/ 


\section{References}

1. Egner T, ed The Wiley Handbook of Cognitive Control: John Wiley \& Sons Ltd. ; 2017.

2. Miller EK, Cohen JD. An integrative theory of prefrontal cortex function. Annual Review of Neuroscience. 2001;24:167-202.

3. Mansouri FA, Tanaka K, Buckley MJ. Conflict-induced behavioural adjustment: a clue to the executive functions of the prefrontal cortex. Nat Rev Neurosci. Feb 2009;10(2):141-152.

4. Stroop JR. Studies of interference in serial verbal reactions. $J$ Exp Psychol. 1935;18:643-662.

5. Botvinick MM, Braver TS, Barch DM, Carter CS, Cohen JD. Conflict monitoring and cognitive control. Psychological Review. Jul 2001;108(3):624-652.

6. Botvinick MM, Cohen JD, Carter CS. Conflict monitoring and anterior cingulate cortex: an update. Trends in Cognitive Sciences. Dec 2004;8(12):539-546.

7. Etkin A, Egner T, Kalisch R. Emotional processing in anterior cingulate and medial prefrontal cortex. Trends in Cognitive Sciences. Feb 2011;15(2):85-93.

8. Critchley HD, Mathias CJ, Josephs O, et al. Human cingulate cortex and autonomic control: converging neuroimaging and clinical evidence. Brain. Oct 2003;126:21392152.

9. Egner T, Hirsch J. Cognitive control mechanisms resolve conflict through cortical amplification of task-relevant information. Nature Neuroscience. Dec 2005;8(12):1784-1790.

10. Ridderinkhof KR, van den Wildenberg WPM, Segalowitz SJ, Carter CS. Neurocognitive mechanisms of cognitive control: The role of prefrontal cortex in action selection, response inhibition, performance monitoring, and reward-based learning. Brain Cognition. Nov 2004;56(2):129-140.

11. Verguts $T$, Notebaert W. Adaptation by binding: a learning account of cognitive control. Trends in Cognitive Sciences. Jun 2009;13(6):252-257.

12. Verguts $T$, Notebaert W. Hebbian learning of cognitive control: Dealing with specific and nonspecific adaptation. Psychological Review. Apr 2008;115(2):518-525.

13. Notebaert W, Verguts T. Cognitive control acts locally. Cognition. Feb 2008;106(2):1071-1080.

14. Unsworth N, Robison MK. A locus coeruleus-norepinephrine account of individual differences in working memory capacity and attention control. Psychon B Rev. Aug 2017;24(4):1282-1311.

15. Unsworth N, Robison MK. The Importance of Arousal for Variation in Working Memory Capacity and Attention Control: A Latent Variable Pupillometry Study. $J$ Exp Psychol Learn. Dec 2017;43(12):1962-1987.

16. Kohler S, Bar KJ, Wagner G. Differential Involvement of Brainstem Noradrenergic and Midbrain Dopaminergic Nuclei in Cognitive Control. Human Brain Mapping. Jun 2016;37(6):2305-2318.

17. Schwarz LA, Miyamichi K, Gao XJJ, et al. Viral-genetic tracing of the input-output organization of a central noradrenaline circuit. Nature. Aug 6 2015;524(7563):88U180.

18. Arnsten AFT, Goldman-Rakic PS. Selective Prefrontal Cortical Projections to the Region of the Locus Coeruleus and Raphe Nuclei in the Rhesus-Monkey. Brain Research. 1984;306(1-2):9-18. 
19. Porrino LJ, Goldman-Rakic PS. Brain-Stem Innervation of Prefrontal and Anterior Cingulate Cortex in the Rhesus-Monkey Revealed by Retrograde Transport of Hrp. $J$ Comp Neurol. 1982;205(1):63-76.

20. Tervo DGR, Proskurin M, Manakov M, et al. Behavioral Variability through Stochastic Choice and Its Gating by Anterior Cingulate Cortex. Cell. Sep 25 2014;159(1):21-32.

21. Chandler DJ, Waterhouse BD, Gao WJ. New perspectives on catecholaminergic regulation of executive circuits: evidence for independent modulation of prefrontal functions by midbrain dopaminergic and noradrenergic neurons. Front Neural Circuit. May 21 2014;8.

22. Waterhouse B, Chandler D, Prouty E, Gao WJ. Electrophysiological Properties of Locus Coeruleus-Prefrontal Cortical Projection Neurons in Normal and Inattentive Rats. Neuropsychopharmacol. Dec 2014;39:S167-S167.

23. Chandler DJ, Gao WJ, Waterhouse BD. Heterogeneous organization of the locus coeruleus projections to prefrontal and motor cortices. P Natl Acad Sci USA. May 6 2014;111(18):6816-6821.

24. Kerns JG, Cohen JD, MacDonald AW, Cho RY, Stenger VA, Carter CS. Anterior Cingulate conflict monitoring and adjustments in control. Science. Feb 13 2004;303(5660):1023-1026.

25. Garavan H, Ross TJ, Murphy K, Roche RAP, Stein EA. Dissociable executive functions in the dynamic control of behavior: Inhibition, error detection, and correction. Neuroimage. Dec 2002;17(4):1820-1829.

26. Koski L, Paus T. Functional connectivity of the anterior cingulate cortex within the human frontal lobe: a brain-mapping meta-analysis. Exp Brain Res. Jul 2000;133(1):55-65.

27. Aston-Jones G, Cohen JD. An integrative theory of locus coeruleus-norepinephrine function: Adaptive gain and optimal performance. Annual Review of Neuroscience. 2005;28:403-450.

28. Hernandez-Perez OR, Hernandez VS, Nava-Kopp AT, et al. A Synaptically Connected Hypothalamic Magnocellular Vasopressin-Locus Coeruleus Neuronal Circuit and Its Plasticity in Response to Emotional and Physiological Stress. Front Neurosci-Switz. Mar 20 2019;13.

29. Cirelli C, Huber R, Gopalakrishnan A, Southard TL, Tononi G. Locus ceruleus control of slow-wave homeostasis. Journal of Neuroscience. May 4 2005;25(18):4503-4511.

30. Redmond DE, Jr., Huang YH. Current concepts. II. New evidence for a locus coeruleus-norepinephrine connection with anxiety. Life Sci. Dec 24 1979;25(26):2149-2162.

31. Weiss JM, Stout JC, Aaron MF, et al. Depression and Anxiety - Role of the LocusCoeruleus and Corticotropin-Releasing Factor. Brain Res Bull. 1994;35(5-6):561-572.

32. Itoi $\mathrm{K}$, Sugimoto $\mathrm{N}$. The brainstem noradrenergic systems in stress, anxiety and depression. J Neuroendocrinol. May 2010;22(5):355-361.

33. McCall JG, Siuda ER, Bhatti DL, et al. Locus coeruleus to basolateral amygdala noradrenergic projections promote anxiety-like behavior. Elife. Jul 14 2017;6.

34. Tanaka M, Yoshida M, Emoto H, Ishii H. Noradrenaline systems in the hypothalamus, amygdala and locus coeruleus are involved in the provocation of anxiety: basic studies. Eur J Pharmacol. Sep 29 2000;405(1-3):397-406.

35. Zhu MY, Klimek V, Dilley GE, et al. Elevated levels of tyrosine hydroxylase in the locus coeruleus in major depression. Biol Psychiatry. Nov 01 1999;46(9):1275-1286.

36. Naegeli C, Zeffiro T, Piccirelli M, et al. Locus Coeruleus Activity Mediates Hyperresponsiveness in Posttraumatic Stress Disorder. Biol Psychiatry. Sep 072017. 
37. Southwick SM, Bremner JD, Rasmusson A, Morgan CA, Arnsten A, Charney DS. Role of norepinephrine in the pathophysiology and treatment of posttraumatic stress disorder. Biol Psychiat. Nov 1 1999;46(9):1192-1204.

38. Etkin A, Egner T, Peraza DM, Kandel ER, Hirsch J. Resolving emotional conflict: A role for the rostral anterior cingulate cortex in modulating activity in the amygdala. Neuron. Sep 21 2006;51(6):871-882.

39. Mayr U, Awh E, Laurey P. Conflict adaptation effects in the absence of executive control. Nature Neuroscience. May 2003;6(5):450-452.

40. Mayr U, Awh E. The elusive link between conflict and conflict adaptation. Psychol Res-Psych Fo. Nov 2009;73(6):794-802.

41. Egner T, Etkin A, Gale S, Hirsch J. Dissociable neural systems resolve conflict from emotional versus nonemotional distracters. Cereb Cortex. Jun 2008;18(6):1475-1484.

42. Robinson OJ, Letkiewicz AM, Overstreet C, Ernst M, Grillon C. The effect of induced anxiety on cognition: threat of shock enhances aversive processing in healthy individuals. Cogn Affect Behav Ne. Jun 2011;11(2):217-227.

43. Braem S, King JA, Korb FM, Krebs RM, Notebaert W, Egner T. The Role of Anterior Cingulate Cortex in the Affective Evaluation of Conflict. J Cognitive Neurosci. Jan 2017;29(1):137-149.

44. Dreisbach G, Fischer R. Conflicts as Aversive Signals for Control Adaptation. Curr Dir Psychol Sci. Aug 2015;24(4):255-260.

45. Egner T. Congruency sequence effects and cognitive control. Cogn Affect Behav Ne. Dec 2007;7(4):380-390.

46. Monti JM, Weintraub S, Egner T. Differential age-related decline in conflict-driven task-set shielding from emotional versus non-emotional distracters. Neuropsychologia. May 2010;48(6):1697-1706.

47. Ashburner J, Friston KJ. Unified segmentation. Neuroimage. Jul 1 2005;26(3):839851.

48. Grueschow M, Polania R, Hare TA, Ruff CC. Automatic versus Choice-Dependent Value Representations in the Human Brain. Neuron. Feb 18 2015;85(4):874-885.

49. Nichols TE, Holmes AP. Nonparametric permutation tests for functional neuroimaging: a primer with examples. Human Brain Mapping. Jan 2002;15(1):1-25.

50. Winkler AM, Ridgway GR, Webster MA, Smith SM, Nichols TE. Permutation inference for the general linear model. Neuroimage. May 15 2014;92:381-397.

51. Keren NI, Lozar CT, Harris KC, Morgan PS, Eckert MA. In vivo mapping of the human locus coeruleus. Neuroimage. Oct 1 2009;47(4):1261-1267.

52. Dimigen O, Valsecchi $M$, Sommer W, Kliegl R. Human Microsaccade-Related Visual Brain Responses. Journal of Neuroscience. Sep 30 2009;29(39):12321-12331.

53. Tse PU, Baumgartner FJ, Greenlee MW. Event-related functional MRI of cortical activity evoked by microsaccades, small visually-guided saccades, and eyeblinks in human visual cortex. Neuroimage. Jan 1 2010;49(1):805-816.

54. Murphy PR, O'Connell RG, O'Sullivan M, Robertson IH, Balsters JH. Pupil diameter covaries with BOLD activity in human locus coeruleus. Human Brain Mapping. Aug 2014;35(8):4140-4154.

55. Murphy PR, Robertson IH, Balsters JH, O'Connell R G. Pupillometry and P3 index the locus coeruleus-noradrenergic arousal function in humans. Psychophysiology. Nov 2011;48(11):1532-1543.

56. de Gee JW, Knapen T, Donner TH. Decision-related pupil dilation reflects upcoming choice and individual bias. P Natl Acad Sci USA. Feb 4 2014;111(5):E618-E625. 
57. Eklund A, Nichols TE, Knutsson H. Cluster failure: Why fMRI inferences for spatial extent have inflated false-positive rates. P Natl Acad Sci USA. Jul 12 2016;113(28):7900-7905.

58. Eklund A, Knutsson $\mathrm{H}$, Nichols TE. Cluster failure revisited: Impact of first level design and physiological noise on cluster false positive rates. Human Brain Mapping. May 2019;40(7):2017-2032.

59. Ridderinkhof KR, Ullsperger M, Crone EA, Nieuwenhuis S. The role of the medial frontal cortex in cognitive control. Science. Oct 15 2004;306(5695):443-447.

60. Krebs RM, Fias W, Achten E, Boehler CN. Picture novelty attenuates semantic interference and modulates concomitant neural activity in the anterior cingulate cortex and the locus coeruleus. Neuroimage. Jul 1 2013;74:179-187.

61. Ide JS, Li CS. A cerebellar thalamic cortical circuit for error-related cognitive control. Neuroimage. Jan 1 2011;54(1):455-464.

62. Chang A, Ide JS, Li HH, Chen CC, Li CSR. Proactive Control: Neural Oscillatory Correlates of Conflict Anticipation and Response Slowing. Eneuro. May-Jun 2017;4(3).

63. Kanwisher N, McDermott J, Chun MM. The fusiform face area: A module in human extrastriate cortex specialized for face perception. Journal of Neuroscience. Jun 1 1997;17(11):4302-4311.

64. Kanwisher N, Yovel G. The fusiform face area: a cortical region specialized for the perception of faces. Philos T R Soc B. Dec 29 2006;361(1476):2109-2128.

65. Heekeren HR, Marrett S, Bandettini PA, Ungerleider LG. A general mechanism for perceptual decision-making in the human brain. Nature. Oct 14 2004;431(7010):859862.

66. Mather M, Clewett D, Sakaki M, Harley CW. Norepinephrine ignites local hot spots of neuronal excitation: How arousal amplifies selectivity in perception and memory. Behavioral and Brain Sciences. Jul 1 2015:1-100.

67. Zhang S, Hu S, Chao HH, Li CS. Resting-State Functional Connectivity of the Locus Coeruleus in Humans: In Comparison with the Ventral Tegmental Area/Substantia Nigra Pars Compacta and the Effects of Age. Cereb Cortex. Aug 2016;26(8):34133427.

68. Kline RL, Zhang S, Farr OM, et al. The Effects of Methylphenidate on Resting-State Functional Connectivity of the Basal Nucleus of Meynert, Locus Coeruleus, and Ventral Tegmental Area in Healthy Adults. Frontiers in Human Neuroscience. 2016;10:149.

69. van der Wel $P$, van Steenbergen $H$. Pupil dilation as an index of effort in cognitive control tasks: A review. Psychon B Rev. Dec 2018;25(6):2005-2015.

70. Brown GG, Kindermann SS, Siegle GJ, Granholm E, Wong EC, Buxton RB. Brain activation and pupil response during covert performance of the Stroop Color Word task. J Int Neuropsych Soc. May 1999;5(4):308-319.

71. Siegle GJ, Steinhauer SR, Thase ME. Pupillary assessment and computational modeling of the Stroop task in depression. Int J Psychophysiol. Mar 2004;52(1):6376.

72. Siegle GJ, Ichikawa N, Steinhauer S. Blink before and after you think: Blinks occur prior to and following cognitive load indexed by pupillary responses.

Psychophysiology. Sep 2008;45(5):679-687.

73. Laeng B, Orbo M, Holmlund T, Miozzo M. Pupillary Stroop effects. Cogn Process. Feb 2011;12(1):13-21. 
74. Joshi S, Li Y, Kalwani RM, Gold JI. Relationships between Pupil Diameter and Neuronal Activity in the Locus Coeruleus, Colliculi, and Cingulate Cortex. Neuron. Jan 6 2016;89(1):221-234.

75. Zerbi V, Floriou-Servou A, Markicevic M, et al. Rapid Reconfiguration of the Functional Connectome after Chemogenetic Locus Coeruleus Activation. Neuron. Aug 21 2019;103(4):702-+.

76. Vinck M, Batista-Brito R, Knoblich U, Cardin JA. Arousal and locomotion make distinct contributions to cortical activity patterns and visual encoding. Neuron. May 6 2015;86(3):740-754.

77. Reimer J, Froudarakis E, Cadwell CR, Yatsenko D, Denfield GH, Tolias AS. Pupil fluctuations track fast switching of cortical states during quiet wakefulness. Neuron. Oct 22 2014;84(2):355-362.

78. McGinley MJ, Vinck M, Reimer J, et al. Waking State: Rapid Variations Modulate Neural and Behavioral Responses. Neuron. Sep 23 2015;87(6):1143-1161.

79. McGinley MJ, David SV, McCormick DA. Cortical Membrane Potential Signature of Optimal States for Sensory Signal Detection. Neuron. Jul 1 2015;87(1):179-192.

80. Niell CM, Stryker MP. Modulation of visual responses by behavioral state in mouse visual cortex. Neuron. Feb 25 2010;65(4):472-479.

81. Constantinople CM, Bruno RM. Effects and mechanisms of wakefulness on local cortical networks. Neuron. Mar 24 2011;69(6):1061-1068.

82. Devilbiss DM, Waterhouse BD. The effects of tonic locus ceruleus output on sensoryevoked responses of ventral posterior medial thalamic and barrel field cortical neurons in the awake rat. Journal of Neuroscience. Dec 1 2004;24(48):10773-10785.

83. Devilbiss DM, Page ME, Waterhouse BD. Locus ceruleus regulates sensory encoding by neurons and networks in waking animals. Journal of Neuroscience. Sep 27 2006;26(39):9860-9872.

84. Rajkowski J, Majczynski H, Clayton E, Aston-Jones G. Activation of monkey locus coeruleus neurons varies with difficulty and performance in a target detection task. $J$ Neurophysiol. Jul 2004;92(1):361-371.

85. Aston-Jones G, Rajkowski J, Kubiak P, Alexinsky T. Locus coeruleus neurons in monkey are selectively activated by attended cues in a vigilance task. Journal of Neuroscience. Jul 1994;14(7):4467-4480.

86. Usher M, Cohen JD, Servan-Schreiber D, Rajkowski J, Aston-Jones G. The role of locus coeruleus in the regulation of cognitive performance. Science. Jan 22 1999;283(5401):549-554.

87. Clayton EC, Rajkowski J, Cohen JD, Aston-Jones G. Phasic activation of monkey locus ceruleus neurons by simple decisions in a forced-choice task. Journal of Neuroscience. Nov 3 2004;24(44):9914-9920.

88. Eason RG, Harter MR, White CT. Effects of Attention and Arousal on Visually Evoked Cortical Potentials and Reaction Time in Man. Physiol Behav. 1969;4(3):283$\&$.

89. Fischer R, Ventura-Bort C, Hamm A, Weymar M. Transcutaneous vagus nerve stimulation (tVNS) enhances conflict-triggered adjustment of cognitive control. Cogn Affect Behav Ne. Aug 2018;18(4):680-693.

90. van Steenbergen H, Band GPH. Pupil dilation in the Simon task as a marker of conflict processing. Frontiers in Human Neuroscience. May 29 2013;7.

91. Laeng B, Orbo M, Holmlund T, Miozzo M. Pupillary Stroop effects. Cognitive Processing. Feb 2011;12(1):13-21.

92. Ide JS, Shenoy P, Yu AJ, Li CS. Bayesian prediction and evaluation in the anterior cingulate cortex. Journal of Neuroscience. Jan 30 2013;33(5):2039-2047. 
93. Hu S, Ide JS, Zhang S, Li CS. Anticipating conflict: Neural correlates of a Bayesian belief and its motor consequence. Neuroimage. Oct 1 2015;119:286-295.

94. Urai AE, Braun A, Donner TH. Pupil-linked arousal is driven by decision uncertainty and alters serial choice bias. Nat Commun. Mar 3 2017;8.

95. Preuschoff K, Hart BM, Einhauser W. Pupil dilation signals surprise: evidence for noradrenaline's role in decision making. Front Neurosci-Switz. 2011;5.

96. Forstmann BU, de Hollander G, van Maanen L, Alkemade A, Keuken MC. Towards a mechanistic understanding of the human subcortex. Nat Rev Neurosci. Jan 2017;18(1):57-65.

97. de Gee JW, Colizoli O, Kloosterman NA, Knapen T, Nieuwenhuis S, Donner TH. Dynamic modulation of decision biases by brainstem arousal systems. Elife. Apr 11 2017;6.

98. Brooks JCW, Faull OK, Pattinson KTS, Jenkinson M. Physiological noise in brainstem fMRI. Frontiers in Human Neuroscience. Oct 4 2013;7.

99. Sasaki M, Shibata E, Tohyama K, et al. Neuromelanin magnetic resonance imaging of locus ceruleus and substantia nigra in Parkinson's disease. Neuroreport. Jul 31 2006;17(11):1215-1218.

100. Astafiev SV, Snyder AZ, Shulman GL, Corbetta M. Comment on "Modafinil Shifts Human Locus Coeruleus to Low-Tonic, High-Phasic Activity During Functional MRI" and "Homeostatic Sleep Pressure and Responses to Sustained Attention in the Suprachiasmatic Area". Science. Apr 16 2010;328(5976).

101. Peterson AC, Li CSR. Noradrenergic Dysfunction in Alzheimer's and Parkinson's Diseases-An Overview of Imaging Studies. Front Aging Neurosci. May 1 2018;10.

102. Hammerer D, Callaghan MF, Hopkins A, et al. Locus coeruleus integrity in old age is selectively related to memories linked with salient negative events. P Natl Acad Sci USA. Feb 27 2018;115(9):2228-2233. 
Figure Legends:
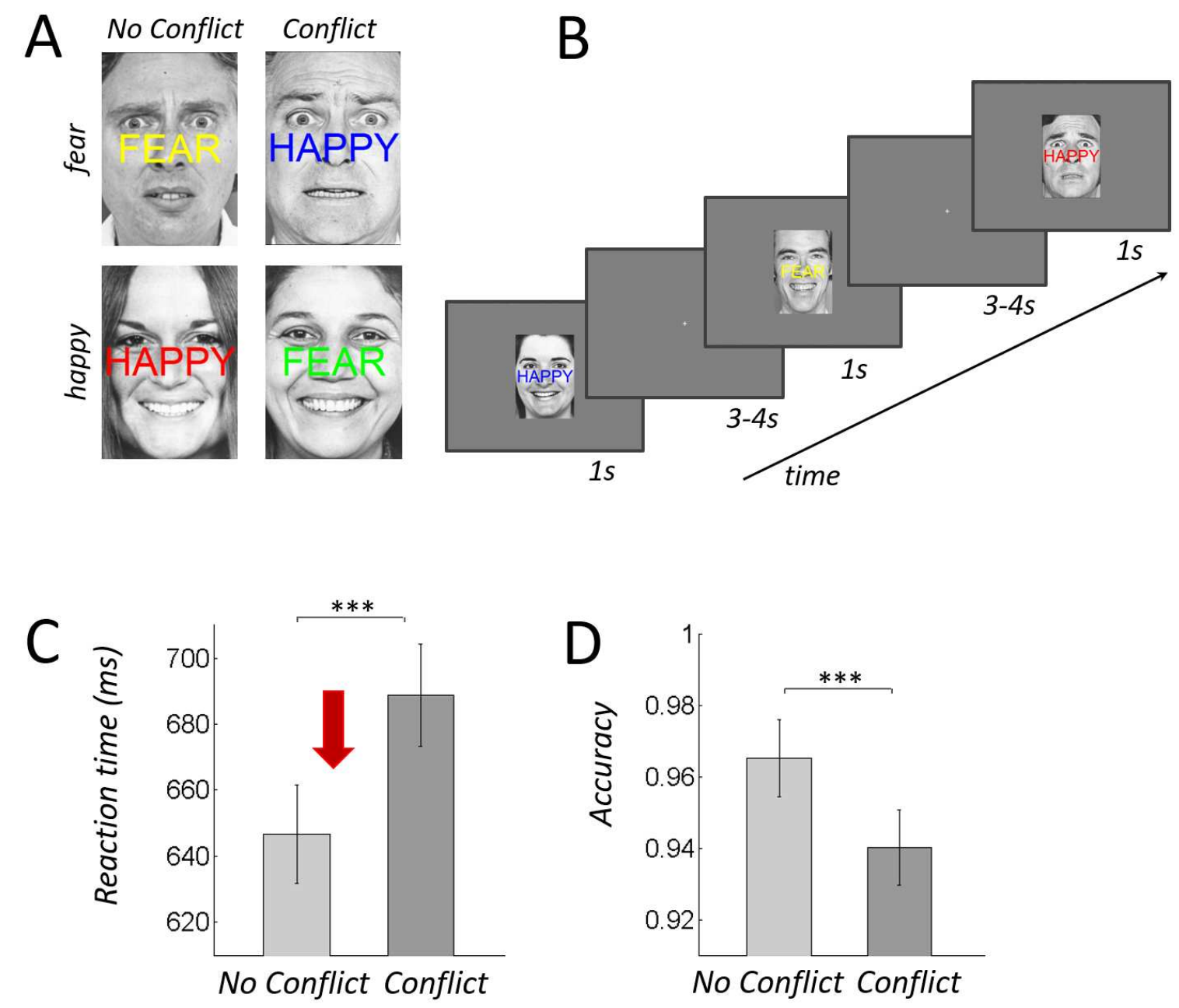

\section{Figure 1. Experimental task and behavioral results.}

(A): Example stimuli illustrating all four possible face/word combinations in the emotional-stroop task. Participants were instructed to react to the facial expression while ignoring the overlaid word and to answer as fast and accurately as possible. On each trial, the word color was randomly assigned in order to avoid adaptation effects. (B): Example trial presentation schedule. (C-D) Conflict trials induce significantly more need for adjustment than co conflict trials, as indicated by (C) increased RTs and (D) decreased accuracy $(\mathrm{N}=48)$. Red arrow in $\mathrm{C}$ indicates the choice conflict adjustment score, which is defiened as difference between RTs in Conflict $>$ No Conflict trials $(\mathrm{I}>\mathrm{C})$. The smaller the individual RT-difference (I>C), the better the participants capacity for choice conflict adjustment and cognitive control. 


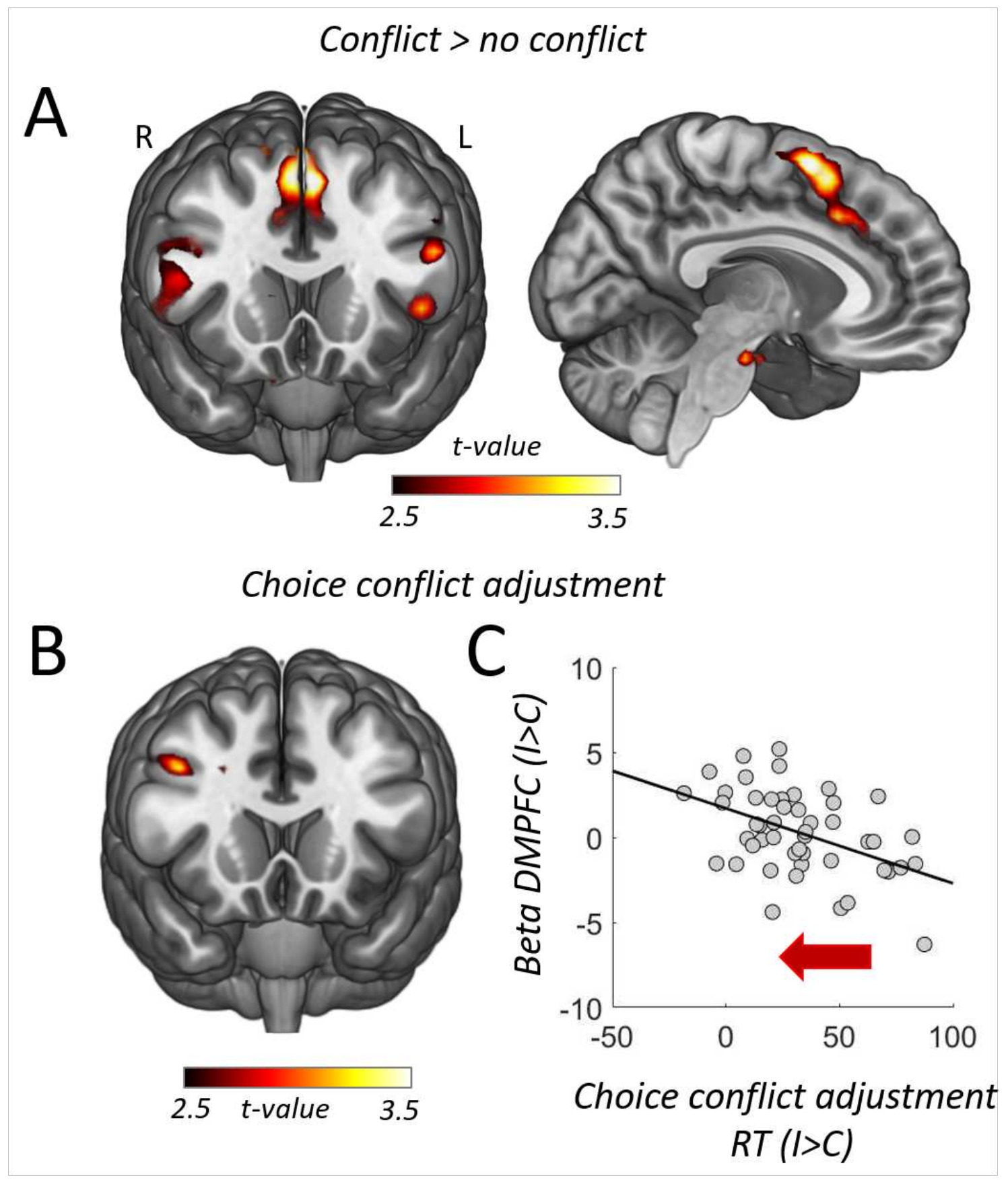

Figure 2. Conflict processing engages the DMPFC, while the level of response conflict resolution is reflected in DLPFC

(A) Response conflict (conflict trials $>$ no conflict trials) activates the dorsomedial prefrontal cortex (DMPFC). (B) The smaller the RT difference between conflict and no conflict trials (the more effective cognitive control), the larger the activation in DLPFC for conflict $>$ no conflict trials. (C) For visualization purposes we plot the correlation between the response conflict resolution score in RT and the BOLD signal differences $(\mathrm{I}>\mathrm{C})$ in the DLPFC coordinates at $\mathrm{X} / \mathrm{Y} / \mathrm{Z}$ : 43/18/35, $(\mathrm{N}=48)$. Neither of 
these effects were observed in the LC-NE using small-volume correction (SVC). Red arrow indicates the response conflict resolution score, defined as the difference between RTs in Conflict $>$ No Conflict trials $(\mathrm{I}>\mathrm{C})$. The smaller the individual RT-difference $(\mathrm{I}>\mathrm{C})$, the better the participant's capacity for response conflict resolution and cognitive control.

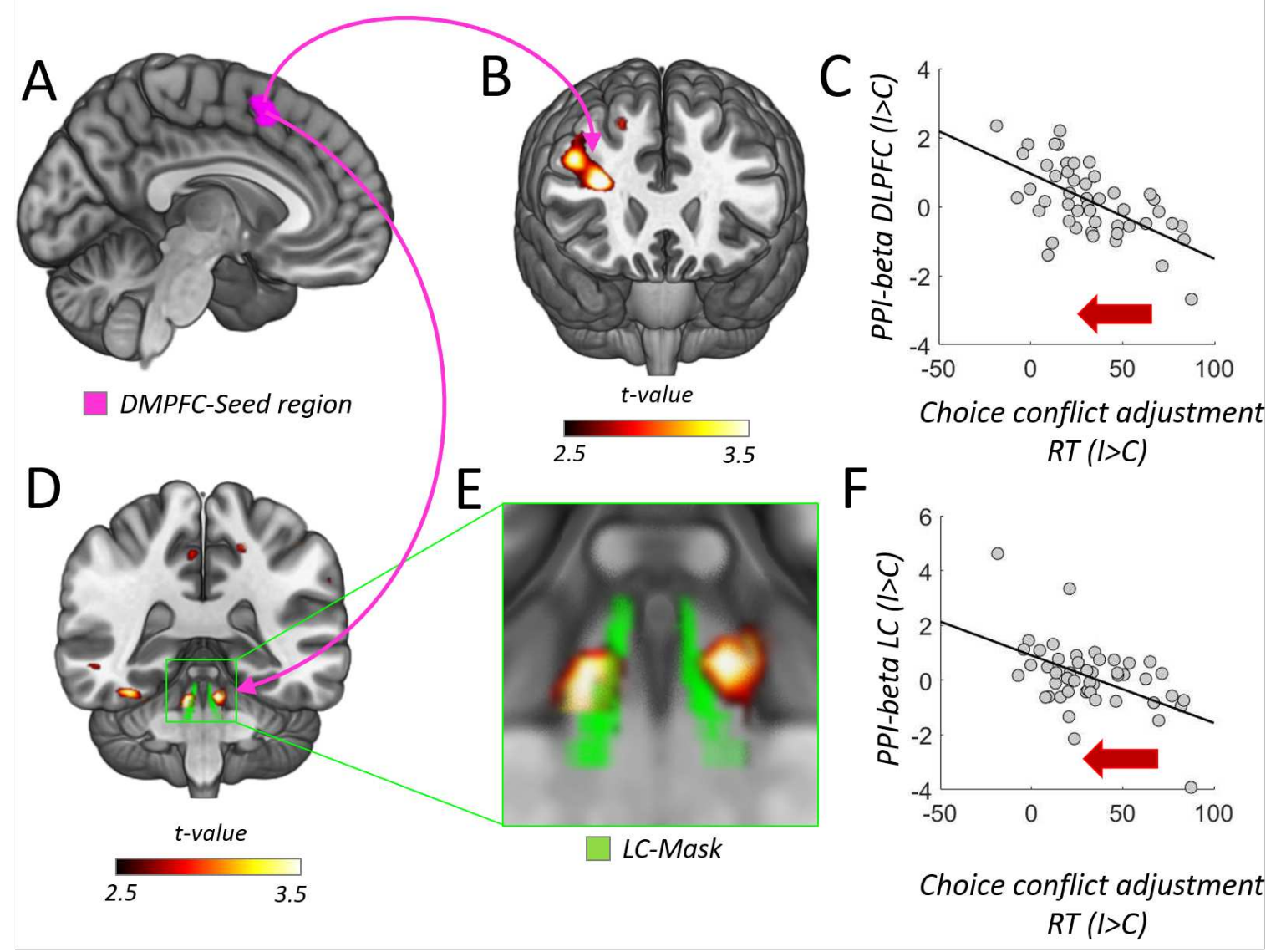

Figure 3. Individual levels of response conflict resolution relate to the strength of functional coupling between DMPFC and DLPFC and LC-NE

(A) DMPFC-seed region for whole brain functional connectivity analysis. (B) The smaller the RT difference between conflict and no conflict trials (i.e., the more effective cognitive control), the larger the functional coupling between DMPFC and DLPFC for conflict $>$ no conflict trials. (C) For visualization purposes: the correlation between the response conflict resolution score in reaction time and the functional coupling between the DMPFC and peak voxel in the DLPFC (voxel coordinates at $\mathrm{X} / \mathrm{Y} / \mathrm{Z}$ : 43/31/35). (D) The smaller the RT difference between incongruent and congruent trials, the larger the functional coupling between DMPFC and LC for conflict $>$ no conflict trials. LC-Mask 
provided by Keren at al. 2009. (E) Enlarged view of the subcortical brainstem, indicating the LC-voxels in the 2SD-mask from Keren et al., 2009 (in green). (F) For visualization purposes: the correlation between the response conflict resolution score in reaction time and the functional coupling between the DMPFC and peak voxel in the locus coeruleus (voxel coordinates at $\mathrm{X} / \mathrm{Y} / \mathrm{Z}$ : 6/-37/-23, $(\mathrm{N}=48)$. Red arrow indicates the response conflict resolution score, defined as the difference between RTs in Conflict $>$ No Conflict trials $(\mathrm{I}>\mathrm{C})$. The smaller the individual RT-difference $(\mathrm{I}>\mathrm{C})$, the better the participants capacity for response conflict resolution and cognitive control.
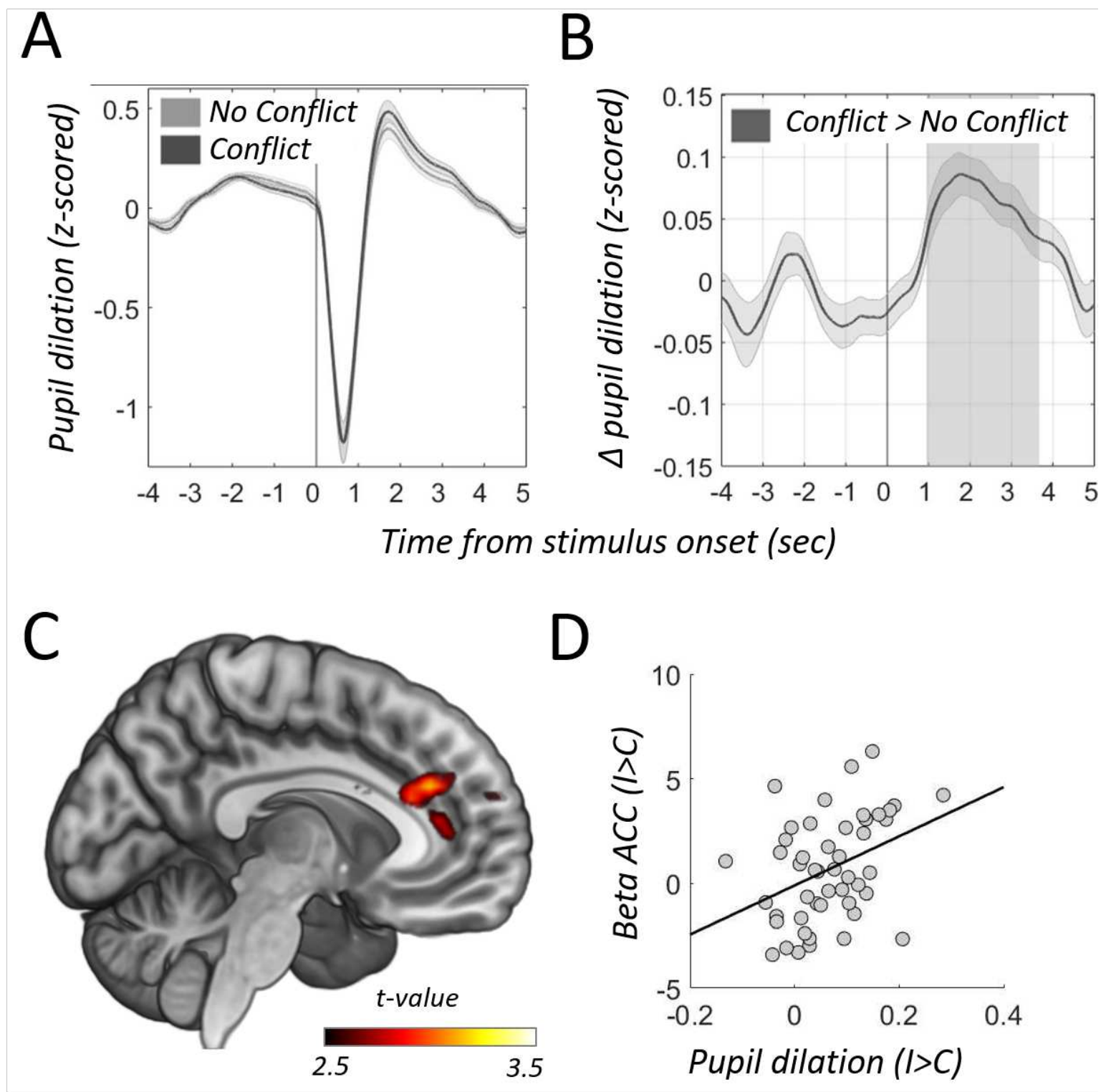

Figure 4. Pupil dilation during response conflict and its relationship to neural conflict signals. 
(A) Mean Pupil dilation during conflict (dark grey) and no conflict trials (light grey). (B) Pupil dilation during conflict trials is significantly larger than during no conflict trials. Grey shades area $=$ sign. difference $\mathrm{p}<0.05$ (cluster corrected). Vertical line $=$ stimulus onset. (C) Pupil-dilation difference between conflict and no conflict trials correlates positively with the BOLD-difference between conflict and no conflict trials in the MPFC/ACC ( $\mathrm{p}=0.001$, uncorrected). (D) For visualization purposes: the correlation between the pupil dilation difference between conflict and no conflict trials $(\mathrm{I}>\mathrm{C})$ and the BOLD activity difference $(\mathrm{I}>\mathrm{C})$ in the anterior cingulate cortex (ACC voxel coordinates at $\mathrm{X} / \mathrm{Y} / \mathrm{Z}$ : $6 / 13 / 25),(N=48)$. 